\title{
On the tails of the limiting distribution function of the error term in the Dirichlet divisor problem
}

\author{
by \\ YUK-KAM LAU (Hong Kong)
}

1. Introduction. Let $d(n)=\sum_{d \mid n} 1$ be the divisor function and define

$$
\Delta(t)=\sum_{n \leq t} d(n)-t(\log t+2 \gamma-1) .
$$

We are concerned with the limiting distribution function $D(\cdot)$ of $t^{-1 / 4} \Delta(t)$, more explicitly,

$$
D(u)=\lim _{T \rightarrow \infty} T^{-1} \mu\left\{t \in[1, T]: t^{-1 / 4} \Delta(t) \leq u\right\}
$$

where $\mu\{\ldots\}$ denotes the Lebesgue measure on $\mathbb{R}$. The existence of $D(u)$ was first established by Heath-Brown [4]. In that paper, he proved that

$$
D(u)=\int_{-\infty}^{u} f(\alpha) d \alpha
$$

where the density function $f(\alpha)$ can be extended to an entire function on the complex plane and satisfies the growth condition

$$
\frac{d^{k}}{d \alpha^{k}} f(\alpha) \ll_{A, k}(1+|\alpha|)^{-A} \text {. }
$$

Concerning the properties of $f(\alpha)$, Heath-Brown [5] gave a further discussion and, in particular, he found that for any $\varepsilon>0$,

$$
f(\alpha) \ll \exp \left(-|\alpha|^{4-\varepsilon}\right) .
$$

Define

$$
\text { tail } D(u)= \begin{cases}D(u) & \text { if } u<0 \\ 1-D(u) & \text { if } u \geq 0\end{cases}
$$

Then (1.2) gives tail $D(u) \ll \exp \left(-|u|^{4-\varepsilon}\right)$. (1.2) can also be shown by Theorem 4.1 of [2] due to Bleher, Cheng, Dyson and Lebowitz; part of their work in [2] is a generalization of [5]. The main focus of [2] is the error term $P_{a}(x)$

2000 Mathematics Subject Classification: Primary 11N60. 
in the shifted circle problem (centered at $a \in \mathbb{R}^{2}$ ). It is proved in [2] that the limiting distribution function $D_{a}(u)$ of the normalized $P_{a}(x)$ satisfies

$$
\exp \left(-|u|^{4+\varepsilon}\right) \ll \text { tail } D_{a}(u) \ll \exp \left(-|u|^{4-\varepsilon}\right) .
$$

Their argument for the lower bound can be applied to the case $\Delta(t)$ as well. So, one can have

$$
\exp \left(-|u|^{4+\varepsilon}\right) \ll \text { tail } D(u) \ll \exp \left(-|u|^{4-\varepsilon}\right) .
$$

Our purpose is to refine (1.3). Throughout the paper, $c, c_{i}$ and $c_{i}^{\prime}(i=$ $1,2, \ldots)$ denote some unspecified positive constants, and the value of $c$ may be different at each occurrence.

Theorem 1. Let $D(u)$ be defined as in (1.1). Then for $|u| \geq 2$,

$$
\exp \left(-c_{1} \frac{|u|^{4}}{(\log |u|)^{\beta}}\right) \ll \operatorname{tail} D(u) \ll \exp \left(-c_{2} \frac{|u|^{4}}{(\log |u|)^{\beta}}\right)
$$

where $\beta=3\left(2^{4 / 3}-1\right) \approx 4.5595$.

This is derived by using the limiting behaviour of the Laplace transform of $D(u)$ at infinity; see [3] and [7] for further information of this approach.

Acknowledgements. The referee points out that Professor Hugh Montgomery has also considered this problem, and has obtained some results (which are as strong as, or even stronger than, the results in this paper), but has not published them. The author wishes to thank the referee for this notification.

2. Some preparations. We need two lemmas to prove our result. Our first lemma is related to Balasubramanian and Ramachandra [1].

Consider a positive multiplicative function $g(n)$ satisfying (i) $g(p)=1 / \gamma$ for all primes $p$, where $\gamma$ is a positive constant and (ii) $g(n) \gg n^{-1 / 16}$. Define $\varepsilon_{k}(n)=1$ if $n$ is $k$-free and 0 otherwise. ( $n$ is $k$-free if there is no prime $p$ such that $p^{k} \mid n$.) Then we have

$$
\sum_{n g(n) \leq x} \varepsilon_{k}(n)=c_{k}^{\prime} x(\log x)^{\gamma-1}+O\left(x(\log x)^{\gamma-11 / 10}\right) .
$$

With $\varepsilon_{k}(n)$ replaced by the constant function 1 , the result (2.1) is proved in [1] (see [1, Theorems 3 and 4]). But our case can be proved in the same way, with the function $f(s)$ in $\left[1\right.$, p. 314] replaced by $f(s)=\sum_{n=1}^{\infty} \varepsilon_{k}(n)(n g(n))^{-s}$. The corresponding $\Phi(s)$ in our situation is

$$
\Phi(s)=f(s) \zeta(s)^{-\gamma^{s}}=\prod_{p}\left(1+\sum_{n=2}^{\infty} c_{n}(s) p^{-n s}\right)
$$


where

$$
c_{n}(s)=\sum_{\substack{r+l=n \\
0 \leq r<k}} \frac{(-1)^{r}}{g\left(p^{l}\right)^{s}}\left(\begin{array}{c}
\gamma^{s} \\
r
\end{array}\right) .
$$

So, $\left|c_{n}(s)\right| \leq p^{n \sigma / 16}\left(1-2^{-\sigma / 16}\right)^{-\gamma^{\sigma}}(\sigma=\operatorname{Re} s)$ and the product in $\Phi(s)$ converges absolutely in $\sigma \geq 3 / 4$.

LEMMA 2.1. Let $g(n)$ and $\varepsilon_{k}(n)$ be defined as above. Then

$$
\sum_{n g(n)>Y} \varepsilon_{k}(n)(n g(n))^{-3 / 2} \asymp Y^{-1 / 2}(\log Y)^{\gamma-1}
$$

and

$$
\sum_{n g(n) \leq Y}(n g(n))^{-3 / 4} \ll Y^{1 / 4}(\log Y)^{\gamma-1} .
$$

Proof. Stieltjes integration with (2.1) gives

$$
\sum_{n g(n)>Y} \varepsilon_{k}(n)(n g(n))^{-3 / 2}=c_{k}^{\prime} \int_{Y}^{\infty} \frac{(\log t)^{\gamma-1}}{t^{3 / 2}} d t+O\left(Y^{-1 / 2}(\log Y)^{\gamma-11 / 10}\right)
$$

and

$$
\sum_{n g(n) \leq Y}(n g(n))^{-3 / 4}=c_{k}^{\prime} \int_{0}^{Y} \frac{(\log t)^{\gamma-1}}{t^{3 / 4}} d t+O\left(Y^{1 / 4}(\log Y)^{\gamma-11 / 10}\right) .
$$

Our result follows.

The next lemma is a weak form of the results in [7] but it is sufficient for our purpose.

LEMMA 2.2. Let $X$ be a real random variable with probability distribution $D(x)$ and let $\phi(x)$ be a regularly varying function with index $0<\alpha<1$, and $\psi(x)$ an asymptotic inverse of $x / \phi(x)$. Suppose that $D(x)>0$ for any $x>0$. There are positive constants $L_{i}, K_{i}(i=1,2,3,4)$ such that

(a) if $\limsup _{\lambda \rightarrow \infty} \psi(\lambda)^{-1} \log E(\exp (-\lambda X)) \leq L_{1}$, then

$$
\limsup _{x \rightarrow \infty} x^{-1} \log D(-\phi(x)) \leq-K_{1}
$$

furthermore, if also $\liminf \operatorname{in}_{\lambda \rightarrow \infty} \psi(\lambda)^{-1} \log E(\exp (-\lambda X)) \geq L_{2}$, then

$$
\liminf _{x \rightarrow \infty} x^{-1} \log D(-\phi(x)) \geq-K_{2}
$$

(b) if $\limsup _{\lambda \rightarrow \infty} \psi(\lambda)^{-1} \log E(\exp (\lambda X)) \leq L_{3}$, then

$$
\limsup _{x \rightarrow \infty} x^{-1} \log (1-D(\phi(x))) \leq-K_{3}
$$

furthermore, if also $\lim \inf _{\lambda \rightarrow \infty} \psi(\lambda)^{-1} \log E(\exp (\lambda X)) \geq L_{4}$, then

$$
\liminf _{x \rightarrow \infty} x^{-1} \log (1-D(\phi(x))) \geq-K_{4} \text {. }
$$


REMARKS. (i) A function $\phi(x)$ is called a regularly varying function with index $\alpha$ if it is a positive measurable function defined for all sufficiently large positive $x$ and $\lim _{x \rightarrow \infty} \phi(\lambda x) / \phi(x)=\lambda^{\alpha}$ for any $\lambda>1$. (Note that this is equivalent to the condition that the limit exists for any $\lambda>0$.)

(ii) $\psi(x)$ is called an asymptotic inverse of $\phi(x)$ if $\lim _{x \rightarrow \infty} \psi(\phi(x)) / x=1$.

Proof of Lemma 2.2. We consider the case (a) only. Its first part follows from [8, Lemma 3.1(b)]. To show the second part, we fix $\xi$ such that $0<$ $\xi<\left(L_{2} / 8\right)^{1 / \alpha}$. Then

$$
\begin{aligned}
E\left(\exp \left(-\frac{\eta}{\phi(\eta)} X\right)\right)= & \int_{-\infty}^{-\phi(\xi \eta)} \exp \left(-\frac{\eta}{\phi(\eta)} x\right) d D(x) \\
& +\int_{-\phi(\xi \eta)}^{\infty} \exp \left(-\frac{\eta}{\phi(\eta)} x\right) d D(x) \\
\leq & \int_{-\infty}^{-\phi(\xi \eta)} \exp \left(-\frac{\eta}{\phi(\eta)} x\right) d D(x)+\exp \left(\eta \frac{\phi(\xi \eta)}{\phi(\eta)}\right) .
\end{aligned}
$$

(Note that $\int_{-\infty}^{\infty} d D(x)=1$.) Since $\liminf _{\lambda \rightarrow \infty} \psi(\lambda)^{-1} \log E(\exp (-\lambda X)) \geq$ $L_{2}$ and $\phi$ is of index $\alpha$, for all large $\eta \geq \eta_{0}=\eta_{0}(\xi)$ we have

$$
\log E\left(\exp \left(-\frac{\eta}{\phi(\eta)} X\right)\right) \geq \frac{L_{2}}{2} \eta \geq 2 \xi^{\alpha} \eta+\log 2 \geq \frac{\phi(\xi \eta)}{\phi(\eta)} \eta+\log 2 .
$$

(Here we need the fact that $\lambda=\eta / \phi(\eta) \rightarrow \infty$ as $\eta \rightarrow \infty$, which is assured by [9, Section 1.1].) Thus, when $\eta \geq \eta_{0}$,

$$
\begin{aligned}
\frac{1}{2} E\left(\exp \left(-\frac{\eta}{\phi(\eta)} X\right)\right) & \leq \int_{-\infty}^{-\phi(\xi \eta)} \exp \left(-\frac{\eta}{\phi(\eta)} x\right) d D(x) \\
& \leq E\left(\exp \left(-2 \frac{\eta}{\phi(\eta)} X\right)\right)^{1 / 2} D(-\phi(\xi \eta))^{1 / 2}
\end{aligned}
$$

by the Cauchy-Schwarz inequality. For any $\varepsilon>0$, we get

$$
2 \eta^{-1} \log E\left(\exp \left(-\frac{\eta}{\phi(\eta)} X\right)\right) \leq \eta^{-1} \log D(-\phi(\xi \eta))+2^{1 /(1-\alpha)} L_{1}+\varepsilon,
$$

for all $\eta \geq \eta_{1}(\varepsilon, \xi)\left(\geq \eta_{0}\right)$. Here we have used

$$
\limsup _{\lambda \rightarrow \infty} \psi(\lambda)^{-1} \log E(\exp (-2 \lambda X)) \leq 2^{1 /(1-\alpha)} L_{1} .
$$

Our result then follows after taking liminf.

Finally, we quote a result [2, Theorem 1.2] about functions satisfying the following hypothesis. 
Hypothesis $\left(\mathrm{H}_{0}\right)$. Let $a_{1}(t), a_{2}(t), \ldots$ be continuous $\mathbb{R}$-valued periodic functions of period 1 such that

$$
\int_{0}^{1} a_{n}(t) d t=0 \quad \text { and } \quad \sum_{n=1}^{\infty} \int_{0}^{1} a_{n}(t)^{2} d t<\infty .
$$

Suppose that there are positive constants $\gamma_{1}, \gamma_{2}, \ldots$ which are linearly independent over $\mathbb{Q}$ such that

$$
\lim _{N \rightarrow \infty} \limsup _{T \rightarrow \infty} \frac{1}{T} \int_{1}^{T} \min \left(1,\left|F(t)-\sum_{n \leq N} a_{n}\left(\gamma_{n} t\right)\right|\right) d t=0 .
$$

THEOREM [BCDL]. Let $F(t)$ satisfy Hypothesis $\left(\mathrm{H}_{0}\right)$. Then for every bounded continuous function $g(x)$ on $\mathbb{R}$,

$$
\lim _{T \rightarrow \infty} \frac{1}{T} \int_{1}^{T} g(F(t)) d t=\int_{-\infty}^{\infty} g(x) \nu(d x)
$$

where $\nu(d x)$ is the distribution of the random series $\eta=\sum_{n=1}^{\infty} a_{n}\left(t_{n}\right)$ and $t_{n}$ 's are independent random variables uniformly distributed on $[0,1]$.

Now take $F(t)=t^{-1 / 2} \Delta\left(t^{2}\right)$ and

$$
a_{n}(t)=\frac{1}{\pi \sqrt{2}} \cdot \frac{\mu(n)^{2}}{n^{3 / 4}} \sum_{r=1}^{\infty} \frac{d\left(n r^{2}\right)}{r^{3 / 2}} \cos (2 \pi r t-\pi / 4)
$$

where $\mu(\cdot)$ is the Möbius function. Moreover, we choose $\gamma_{n}=2 \sqrt{n}$ for squarefree $n$, and for other $n$ we take any positive numbers such that $\left\{\gamma_{1}, \gamma_{2}, \ldots\right\}$ is linearly independent over $\mathbb{Q}$. Then, by Heath-Brown's result and Theorem [BCDL], we see that $D(u)$ (defined in (1.1)) is the probability distribution of the random variable $X=\sum_{n=1}^{\infty} a_{n}\left(t_{n}\right)$ where $t_{n}$ 's are independent random variables uniformly distributed on $[0,1]$. (Note that the infinite sum converges almost surely by Kolmogorov's theorem.)

3. Proof of Theorem 1. Since $X=\sum_{n=1}^{\infty} a_{n}\left(t_{n}\right)$ and $t_{n}$ 's are independent random variables uniformly distributed on $[0,1]$, we have

$$
E(\exp ( \pm \lambda X))=\prod_{n=1}^{\infty} E\left(\exp \left( \pm \lambda a_{n}(t)\right)\right)=\prod_{n=1}^{\infty} \int_{0}^{1} \exp \left( \pm \lambda a_{n}(t)\right) d t
$$

Our task is to bound $\log E(\exp ( \pm \lambda X))$ from above and below for $\lambda \geq 1$. We first recall some simple inequalities: $e^{x} \leq 1+x+x^{2}$ if $x \leq 1, e^{x} \geq 1+x$ and $e^{x} \geq 1+x+x^{2} / 2+x^{3} / 6$ for all $x$. Also, for squarefree $n$, we have

$$
\left|a_{n}(t)\right| \leq c d(n) n^{-3 / 4}
$$




$$
\begin{aligned}
& \int_{0}^{1} a_{n}(t)^{2} d t=\frac{\mu(n)^{2}}{4 \pi^{2} n^{3 / 2}} \sum_{r=1}^{\infty} \frac{d\left(n r^{2}\right)^{2}}{r^{3}} \asymp\left(\frac{d(n)}{n^{3 / 4}}\right)^{2} \\
& \int_{0}^{1} a_{n}(t)^{3} d t=\frac{3 \mu(n)^{2}}{16 \pi^{3} n^{9 / 4}} \sum_{r, s=1}^{\infty} \frac{d\left(n r^{2}\right) d\left(n s^{2}\right) d\left(n(r+s)^{2}\right)}{r^{3 / 2} s^{3 / 2}(r+s)^{3 / 2}} \ll\left(\frac{d(n)}{n^{3 / 4}}\right)^{3}
\end{aligned}
$$

where all the implied constants are absolute. Let $\varepsilon_{0}>0$ be sufficiently small. When $\lambda d(n) / n^{3 / 4}<\varepsilon_{0}$, from $\int_{0}^{1} a_{n}(t) d t=0$ we have

$$
\begin{aligned}
1+\frac{\lambda^{2}}{4} \int_{0}^{1} a_{n}(t)^{2} d t & \leq 1 \pm \lambda \int_{0}^{1} a_{n}(t) d t+\frac{\lambda^{2}}{2} \int_{0}^{1} a_{n}(t)^{2} d t \pm \frac{\lambda^{3}}{6} \int_{0}^{1} a_{n}(t)^{3} d t \\
& \leq \int_{0}^{1} \exp \left( \pm \lambda a_{n}(t)\right) d t \leq 1+\lambda^{2} \int_{0}^{1} a_{n}(t)^{2} d t
\end{aligned}
$$

Thus, as $\log (1+y) \leq y$ for $y \geq 0$ and $\log (1+y) \geq y / 2$ if $0 \leq y \leq 1$, we get

$$
\frac{\lambda^{2}}{8} \int_{0}^{1} a_{n}(t)^{2} d t \leq \log \int_{0}^{1} \exp \left( \pm \lambda a_{n}(t)\right) d t \leq \lambda^{2} \int_{0}^{1} a_{n}(t)^{2} d t .
$$

If $\lambda d(n) / n^{3 / 4} \geq \varepsilon_{0}$, then

$$
1=\int_{0}^{1}\left(1 \pm \lambda a_{n}(t)\right) d t \leq \int_{0}^{1} \exp \left( \pm \lambda a_{n}(t)\right) d t \leq \exp \left(c \lambda \frac{d(n)}{n^{3 / 4}}\right)
$$

Hence,

$$
\lambda^{2} \sum_{\lambda d(n) / n^{3 / 4}<\varepsilon_{0}} \mu(n)^{2}\left(\frac{d(n)}{n^{3 / 4}}\right)^{2} \ll \log E(\exp ( \pm \lambda X))
$$

and

$$
\log E(\exp ( \pm \lambda X)) \ll \lambda^{2} \sum_{\lambda d(n) / n^{3 / 4}<\varepsilon_{0}} \mu(n)^{2}\left(\frac{d(n)}{n^{3 / 4}}\right)^{2}+\lambda \sum_{\lambda d(n) / n^{3 / 4} \geq \varepsilon_{0}} \frac{d(n)}{n^{3 / 4}}
$$

where the implied constants depend on $\varepsilon_{0}$. Now, we take

$$
\phi(x)=\left(x \log ^{3(\gamma-1)} x\right)^{1 / 4}, \quad \psi(x)=x^{4 / 3} \log ^{\gamma-1} x \quad \text { where } \quad \gamma=2^{4 / 3} .
$$

Applying Lemma 2.1 with $g(n)=d(n)^{-4 / 3}$ and $Y=\left(\varepsilon_{0}^{-1} \lambda\right)^{4 / 3}$ (note that $d(n) \ll n^{\varepsilon}$ and so $\left.g(n) \gg n^{-\varepsilon}\right)$, we see that $\log E(\exp ( \pm \lambda X)) \asymp \lambda^{4 / 3}(\log \lambda)^{\gamma-1}$ $=\psi(\lambda)$. Our result follows from Lemma 2.2 .

4. Final remark. The above argument can be applied to $E(t), \Delta_{3}(t)$ and $P(t)$ which are the error terms in the mean square formula of the Riemann zeta-function on the critical line, in the Piltz divisor problem and the circle problem respectively. 
Theorem 2. Write $D_{h}(u)=\lim _{T \rightarrow \infty} T^{-1} \mu\{t \in[1, T]: h(t) \leq u\}$ for the limiting distribution function of a real-valued function $h$. Then

1. when $h(t)=t^{-1 / 4} E(t)$, (1.4) holds with $D(u)$ replaced by $D_{h}(u)$;

2. when $h(t)=t^{-1 / 3} \Delta_{3}(t)$, we have, with $\kappa=2\left(3^{3 / 2}-1\right)$,

$$
\exp \left(-c_{3}|u|^{3} /(\log |u|)^{\kappa}\right) \ll \operatorname{tail} D_{h}(u) \ll \exp \left(-c_{4}|u|^{3} /(\log |u|)^{\kappa}\right) ;
$$

3. when $h(t)=t^{-1 / 4} P(t)$, we have, with $\theta=3\left(2^{1 / 3}-1\right)$,

$$
\exp \left(-c_{5}|u|^{4} /(\log |u|)^{\theta}\right) \ll \operatorname{tail} D_{h}(u) \ll \exp \left(-c_{6}|u|^{4} /(\log |u|)^{\theta}\right) .
$$

Proof. For $E(t)$, we take $\gamma_{n}=\sqrt{2 n / \pi}$ when $n$ is squarefree and any suitable value otherwise; and the function $a_{n}(t)$ is given by

$$
a_{n}(t)=\left(\frac{2}{\pi}\right)^{1 / 4} \frac{\mu(n)^{2}}{n^{3 / 4}} \sum_{r=1}^{\infty}(-1)^{n r} \frac{d\left(n r^{2}\right)}{r^{3 / 2}} \cos (2 \pi r t-\pi / 4) .
$$

(See $\left[4\right.$, Section 6].) It is clear from the proof that the factor $(-1)^{n r}$ will not affect the argument and hence the result.

In the case of $\Delta_{3}(t)$, from [4, Section 7], we choose $\gamma_{n}=n^{1 / 3}$ when $\varepsilon_{3}(n)=1$ and

$$
a_{n}(t)=\frac{1}{\pi \sqrt{3}} \cdot \frac{\varepsilon_{3}(n)}{n^{2 / 3}} \sum_{r=1}^{\infty} \frac{d_{3}\left(n r^{3}\right)}{r^{2}} \cos (6 \pi r t) .
$$

Accordingly, from (2.1) we obtain

$$
\begin{aligned}
\lambda^{2} \sum_{\lambda d_{3}(n) / n^{2 / 3}<\varepsilon_{0}} \varepsilon_{3}(n)\left(\frac{d_{3}(n)}{n^{2 / 3}}\right)^{2} & \ll \log E(\exp ( \pm \lambda X)) \\
\ll & \lambda^{2} \sum_{\lambda d_{3}(n) / n^{2 / 3}<\varepsilon_{0}} \varepsilon_{3}(n)\left(\frac{d_{3}(n)}{n^{2 / 3}}\right)^{2} \\
& +\lambda \sum_{\lambda d_{3}(n) / n^{2 / 3} \geq \varepsilon_{0}} \varepsilon_{3}(n) \frac{d_{3}(n)}{n^{2 / 3}} .
\end{aligned}
$$

We see that both sides are $\asymp \lambda^{3 / 2}(\log \lambda)^{\varrho}$ where $\varrho=3^{3 / 2}-1$. Taking $\psi(\lambda)=$ $\lambda^{3 / 2}(\log \lambda)^{\varrho}$ and $\phi(x)=x^{1 / 3}(\log x)^{2 \varrho / 3}$ and applying Lemma 2.2 yields the result.

Finally, from [4, Section 6] again, the choice of $\gamma_{n}$ for $P(t)$ is $\sqrt{n}(n$ squarefree) and

$$
a_{n}(t)=-\frac{1}{\pi} \cdot \frac{\mu(n)^{2}}{n^{3 / 4}} \sum_{l=1}^{\infty} \frac{r\left(n l^{2}\right)}{l^{3 / 2}} \cos (2 \pi l t+\pi / 4) .
$$


From [6, Section 6.7], we know that $\delta(n)=r(n) / 4$ is multiplicative and

$$
\frac{r\left(p^{m}\right)}{4}= \begin{cases}1 & \text { if } p=2 \\ \left(1+(-1)^{m}\right) / 2 & \text { if } p \equiv 3(\bmod 4) \\ m+1 & \text { if } p \equiv 1(\bmod 4)\end{cases}
$$

Hence $\delta\left(p^{m+2 k}\right) \leq \delta\left(p^{m}\right) \delta\left(p^{2 k}\right)$. Together with $r(n) \ll n^{\varepsilon}$, we obtain $r(n) \leq$ $r\left(n l^{2}\right) \ll l^{\varepsilon} r(n)$, and therefore,

$$
\begin{aligned}
\lambda^{2} \sum_{\lambda r(n) / n^{3 / 4}<\varepsilon_{0}} \mu(n)^{2}\left(\frac{r(n)}{n^{3 / 4}}\right)^{2} & \ll \log E(\exp ( \pm \lambda X)) \\
\ll & \lambda^{2} \sum_{\lambda r(n) / n^{3 / 4}<\varepsilon_{0}} \mu(n)^{2}\left(\frac{r(n)}{n^{3 / 4}}\right)^{2} \\
& +\lambda \sum_{\lambda r(n) / n^{3 / 4} \geq \varepsilon_{0}} \mu(n)^{2} \frac{r(n)}{n^{3 / 4}}
\end{aligned}
$$

We now need an asymptotic formula for $\sum_{n / r(n)^{4 / 3} \leq x} \mu(n)^{2}$ where the sum is restricted to $r(n)>0$. Similarly to the proof of $(2.1)$, we consider $f(s)=\sum_{n=1}^{\infty} \mu(n)^{2} r(n)^{4 s / 3} n^{-s} ;$ (4.1) yields that

$$
f(s)=4^{4 s / 3}\left(1+2^{-s}\right) \prod_{p \equiv 1(4)}\left(1+2^{4 s / 3} p^{-s}\right) .
$$

Define the Dirichlet character $\chi_{4}(n)=1$ or -1 according as $n \equiv 1$ or $3(\bmod$ $4)$, and $\chi_{4}(n)=0$ otherwise. The associated $L$-function is

$$
L\left(s, \chi_{4}\right)=\prod_{p \equiv 1(4)}\left(1-p^{-s}\right)^{-1} \prod_{p \equiv 3(4)}\left(1+p^{-s}\right)^{-1}
$$

for $\operatorname{Re} s>1$. Thus, we have

$$
\zeta(s) L\left(s, \chi_{4}\right)=\left(1-2^{-s}\right)^{-1} \prod_{p \equiv 1(4)}\left(1-p^{-s}\right)^{-2} \prod_{p \equiv 3(4)}\left(1-p^{-2 s}\right)^{-1} .
$$

Define

$$
\begin{aligned}
\Phi(s)= & f(s)\left(\zeta(s) L\left(s, \chi_{4}\right)\right)^{-2^{(4 s-3) / 3}} \\
= & 4^{4 s / 3}\left(1+2^{-s}\right)\left(1-2^{-s}\right)^{2^{(4 s-3) / 3}} \prod_{p \equiv 3(4)}\left(1-p^{-2 s}\right)^{2^{(4 s-3) / 3}} \\
& \times \prod_{p \equiv 1(4)}\left(1+2^{4 s / 3} p^{-s}\right)\left(1-p^{-s}\right)^{2^{4 s / 3}} .
\end{aligned}
$$

Then $\Phi(s)$ is holomorphic on the half-plane $\operatorname{Re} s \geq 1-\varepsilon_{0}$ for some small 
constant $\varepsilon_{0}>0$. Repeating the argument of [1], we obtain

$$
\sum_{n / r(n)^{4 / 3} \leq x} \mu(n)^{2}=c x(\log x)^{\alpha}+O\left(x(\log x)^{\alpha-\varepsilon}\right)
$$

with $\alpha=2^{1 / 3}-1$. By partial summation, (4.2) yields $\log E(\exp ( \pm \lambda X)) \asymp$ $\lambda^{4 / 3}(\log \lambda)^{\alpha}$ which will be our $\psi(\lambda)\left(\operatorname{so} \phi(x)=x^{1 / 4}(\log x)^{3 \alpha / 4}\right)$.

\section{References}

[1] R. Balasubramanian and K. Ramachandra, On the number of integers $n$ such that $n d(n) \leq x$, Acta Arith. 49 (1988), 313-322.

[2] P. M. Bleher, Z. Cheng, F. J. Dyson and J. L. Lebowitz, Distribution of the error term for the number of lattice points inside a shifted circle, Comm. Math. Phys. 154 (1993), 433-469.

[3] T. Hattori and K. Matsumoto, A limit theorem for Bohr-Jessen's probability measures of the Riemann zeta-function, J. Reine Angew. Math. 507 (1999), 219-232.

[4] D. R. Heath-Brown, The distribution and moments of the error term in the Dirichlet divisor problem, Acta Arith. 60 (1992), 389-415.

[5] - , The Dirichlet divisor problem, in: Advances in Number Theory, F. Q. Gouvêa and N. Yui (eds.), Clarendon Press, Oxford, 1993, 31-35.

[6] L. K. Hua, Introduction to Number Theory, Springer, 1982.

[7] Y. Kasahara, Tauberian theorems of exponential type, J. Math. Kyoto Univ. 18 (1978), 209-219.

[8] Y. K. Lau, On the limiting distribution of a generalized divisor problem for the case $-1 / 2<a<0$, Acta Arith. 98 (2001), 229-236.

[9] E. Seneta, Regularly Varying Functions, Lecture Notes in Math. 508, Springer, 1976.

Department of Mathematics

The University of Hong Kong

Pokfulam Road, Hong Kong

E-mail: yklau@maths.hku.hk

Received on 27.10.1999

and in revised form on 23.4.2001 